\title{
RESPUESTA DE LA COMUNIDAD DE PECES AL DRAGADO DE RIOS COSTEROS DE LA REGION DEL BIOBIO (CHILE)
}

\section{RESPONSE OF THE FISH COMMUNITY TO DREDGE OF COASTAL RIVERS IN THE BIOBIO REGION (CHILE)}

\author{
Juan J. Ortiz-Sandoval ${ }^{1}$, Néstor Ortiz ${ }_{1}^{1}$, Roberto Cifuentes ${ }_{1}^{2,1}$, \\ Jorge González ${ }^{1} \&$ Evelyn Habit ${ }^{1}$ \\ Unidad de Sistemas Acuáticos, Centro de Ciencias Ambientales EULA-Chile, Universidad de Concepción. Barrio \\ $2 \quad$ Universitario s/n, Concepción, Chile. \\ Departamento de Zoología, Facultad de Ciencias Naturales y Oceanográficas, Universidad de Concepción. Barrio \\ Universitario s/n, Concepción, Chile.
}

juanorti@udec.cl

\section{RESUMEN}

Las comunidades de peces de agua dulce presentan grados de resistencia y resiliencia a actividades antrópicas, los cuales se relacionan positivamente con su diversidad específica y negativamente con los niveles de intervención previa. Los ríos costeros de la zona Centro-Sur de Chile se caracterizan por albergar una diversa ictiofauna, principalmente en sus zonas bajas. En particular, el sector de potamón de los ríos Andalién y Nonguén, ambos insertos en la ciudad de Concepción, son de alto valor de conservación de su biodiversidad. En estos sectores, los eventos recurrentes de inundación de zonas residenciales, construidas en las planicies de inundación, han llevado a que ambos cauces sean dragados y semicanalizados. Las respuestas de las comunidades de peces a este tipo de intervención no han sido estudiadas en Chile. Postulamos que dada su alta diversidad, y bajo nivel de intervención previa, las comunidades ícticas de los ríos Andalién y Nonguén son resistentes y resilientes a los efectos del dragado. Para entender la respuesta comunitaria en ambos ríos, en este estudio primero analizamos los cambios en la estructura en las situaciones previo al dragado, con acciones de dragado y posterior a las mismas. En segundo lugar, analizamos las respuestas especie-específicas, para explicar los cambios comunitarios y conocer la sensibilidad de las distintas especies a esta intervención. Inmediatamente después del dragado se produjo una disminución de la diversidad específica, generándose la dominancia de una única especie, Cheirodon galusdae. Posteriormente, se evidenció una recuperación parcial de los parámetros comunitarios. Basados en las respuestas especie-específicas a este tipo de perturbación identificamos 3 especies resistentes, 3 especies resilientes y 8 especies sensibles. De estas últimas, Nematogenys inermis es la de mayor relevancia en términos de conservación.

Palabras Claves: Nematogenys inermis, peces nativos, respuesta comunitaria, Río Andalién, Río Nonguén.

\section{ABSTRACT}

The freshwater fish communities present degrees of resistance and resilience to human activities, which have a positive relationship with species diversity, and a negative relation with levels of previous intervention. The costal rivers in the Central-Southern zone of Chile are characterized by a diverse icthyofauna, principally in the low zones. Particularly, the potamon areas of the Andalién and Nonguén rivers, both running through the city of Concepción, have a high conservation value of biodiversity. In these areas, the reoccurring flooding in residential zones constructed over the floodplain has caused both channels to be dredged and semi-canalized. The fish community's response to this type of interventions has not been studied yet in Chile. We propose, given its high diversity and low level of previous intervention, that the fish community of the Andalién and Nonguén rivers is resilient to the effects of the dredge. To understand the community response in both rivers, we first analyzed the changes in the structure in situations before the dredge, with dredge actions, as well as after the dredge. Second, we analyzed the species-specific response to explain the community changes and be able to understand the sensibility of the different species to this intervention. Immediately after the dredge, a decrease of the species diversity occurred, generating the dominance of a single species, Cheirodon galusdae. Later, a 
Respuesta de la comunidad de peces al dragado: OrTIZ-SANDOval, J. ET AL.

partial recovery of the community parameters was noted. Based on species-specific responses of this type of perturbation, we identified 3 resistant species, 3 resilient species and 8 sensitive species. Among these last species, Nematogenys inermis has the highest relevance in conservation terms.

KEYwords: Nematogenys inermis, native icthyofauna, community response, Andalien River, Nonguen River.

\section{INTRODUCCION}

Las comunidades de peces de sistemas fluviales presentan patrones de zonación característicos de incremento de la riqueza de especies, abundancia de individuos y tallas corporales en el sentido de la corriente (Welcomme 1985). Ello resulta particularmente evidente en las comunidades de peces en ríos chilenos, los cuales tienden a ser cortos y de alta pendiente (Campos 1985). En éstos, tanto la riqueza específica como la abundancia tienden a aumentar desde las zonas de cabecera o ritrales a las zonas bajas o potamales (Duarte et al. 1971, Campos 1985, Campos et al. 1993, Ruiz 1996, Habit et al. 2006a). De esta forma, las zonas bajas de los ríos chilenos son también las áreas de más alto valor de conservación para la ictiofauna nativa, ya que albergan la mayor diversidad de especies (Soto $e t$ al. 2006, Habit et al. 2007a). Por otra parte, tales zonas son las que presentan mayor intervención humana, manifestándose en ellas los efectos acumulados o sinérgicos de las actividades que se desarrollan a lo largo de la cuenca hidrográfica (Illies $\&$ Botaseanu 1963). Esto impone un gran desafío para la conservación de la ictiofauna nativa dulceacuícola en Chile, ya que su mayor diversidad ocurre en las áreas de más alta intervención. Ello implica conciliar las diferentes actividades necesarias para el desarrollo humano (extracción de agua, vertimiento de efluentes, extracción de áridos, entre otras) con la mantención de las especies acuáticas que sustentan el funcionamiento de los ecosistemas y que son valiosas en términos de biodiversidad (Dudgeon 2000). En este contexto, la ictiofauna nativa chilena de aguas continentales es de alto valor de conservación, ya que se caracteriza por su baja riqueza de especies, alto porcentaje de endemismo y por retener características primitivas (Vila et al. 1999, Dyer 2000, Habit et al 2006b).

De los sistemas fluviales estudiados en Chile, la ictiofauna del río Andalién (Región del Biobío, área centro-sur de endemismo sensu Dyer 2000) ha sido citada como una de las más diversas a pesar del reducido tamaño de su cuenca hidrográfica (Ruiz 1993, Habit \& Victoriano 2005). Dicha ictiofauna presenta un claro patrón de incremento de la diversidad específica en el sentido de la corriente, el cual no resulta del reemplazo de especies a lo largo del gradiente ambiental, sino de la acumulación de nuevas especies a lo largo del mismo (Habit et al. 2007a). De esta forma, la ictiofauna de las zonas ritrales y transicionales representa un subgrupo anidado de la comunidad de peces que habita en la zona potamal, la cual resulta más rica y abundante. En esta cuenca, las zonas bajas, principalmente de los ríos Andalién propiamente tal y Nonguén (tributario del anterior) se ubican directamente en la zona urbana de Concepción (Fig. 1), y presentan alteraciones en su calidad del agua debido al incremento de materia orgánica y coliformes fecales (Jaque 1994, Habit et al. 2003), lo cual no afecta severamente los patrones comunitarios de la ictiofauna (Habit et al. 2007a). Sin embargo, las inundaciones de extensos sectores de la ciudad construidos sobre las planicies de inundación, han derivado en intensivas acciones de dragado y semicanalización de los sectores potamales de ambos ríos. Estas intervenciones directas en el cauce tienen por objetivo ensanchar y profundizar el canal principal de los ríos. Las respuestas de las comunidades de peces a este tipo de perturbaciones no han sido estudiadas en Chile. Hasta ahora, se ha reportado que las comunidades de peces de ríos Andinos de la misma región, muestran respuestas diferenciales a perturbaciones antrópicas como la construcción y operación de centrales hidroeléctricas, según el grado de intervención previa del sistema fluvial, resultando de mayor resistencia y resiliencia las comunidades con mayor riqueza específica que habitan ambientes con menor grado de intervención antrópica previa (Habit et al. 2007b). Igualmente, se han evidenciado efectos combinados de diversas actividades antrópicas tales como la regulación de caudales por la operación de centrales y la contaminación química por efluentes de celulosa. Los que alteran los patrones de distribución longitudinales descritos para la ictiofauna de ríos chilenos (Habit et al. 2006a). Considerando que la ictiofauna de los ríos Andalién y Nonguén es diversa y que ambos ríos 
no presentan altos grados de deterioro ambiental previo al dragado, postulamos que esta intervención genera cambios comunitarios transitorios, y que las comunidades tienden a recuperar su estado previo. Para analizar la respuesta comunitaria, analizamos el efecto de las obras de dragado efectuadas en los cauces de lo ríos Andalién y Nonguén en el año 2006, bajo dos perspectivas. Primero, dado que las historias de vida de las diferentes especies pueden generar respuestas diferenciales, analizamos las respuestas especie-específicas al dragado. En segundo lugar, analizamos los cambios en la estructura comunitaria en las situaciones previo al dragado, con acciones de dragado y posterior a las mismas. Comprender el significado de estas acciones, es de gran importancia para la conservación de la valiosa fauna íctica que habitan estos sistemas fluviales costeros.

\section{MATERIALES Y METODOS}

\section{AREA DE ESTUDIO}

La cuenca del río Andalién ( $\left.36^{\circ} 45^{`} \mathrm{~S}, 72^{\circ} 50^{`} \mathrm{~W}\right)$ tiene su origen en la Cordillera de la Costa, abarcando un total de 770 kilómetros cuadrados y alcanzando un orden 4 en su desembocadura. La zona potamal del río Andalién propiamente tal y su último tributario, el río Nonguén, se caracterizan por una baja profundidad $(<50 \mathrm{~cm})$, sustrato de gravilla y arena gruesa, y abundante vegetación ribereña de matorral. Estos sectores fueron dragados durante agosto y septiembre del 2006 con el propósito de ensanchar y profundizar sus cauces. Inmediatamente después del dragado, ambos cursos de agua aumentaron en promedio $30 \mathrm{~cm}$ su profundidad, $10 \mathrm{~m}$ de ancho y perdieron la vegetación ribereña en una franja de $3 \mathrm{~m}$ promedio. Posteriormente, existió una recuperación parcial de la vegetación.

\section{MÉTOdOS}

La ictiofauna de la zona potamal de la cuenca del río Andalién fue estudiada mediante el muestreo de una estación en la zona baja del río Andalién propiamente tal (P1) y una en el río Nonguén (P2), tributario inferior del Andalién (Fig. 1). Para analizar las respuestas especie específicas y comunitaria al dragado de ambos ríos, se compararon la abundancia, riqueza específica y diversidad de la ictiofauna en las situaciones previa al dragado o situación "SIN" (2004), inmediatamente después del dragado o situación "CON" (2006) y en la situación post-dragado de recuperación o "POST" (2007). Los datos correspondientes al año 2004 fueron obtenidos de Habit et al. (2007a), utilizando la abundancia promedio obtenida en cuatro muestreos estacionales en las mismas estaciones de muestreo, las que no mostraron diferencias temporales estadísticamente significativas. Las muestras de la situación "CON" fueron obtenidas en octubre de 2006, a un mes de los principales eventos de dragado. Las muestras de la situación "POST" fueron obtenidas en marzo de 2007, es decir 6 meses de la intervención. Todos los muestreos fueron estandarizados y consistieron en la remoción de todos los peces encontrados en un segmento de $100 \mathrm{~m}$ de río mediante el uso de un equipo de pesca eléctrica Elektrofishfangerat motor JLO a gasolina, 50-400 volts, DC de un ánodo. Los individuos capturados fueron identificados a nivel de especie in situ, cuantificados y devueltos a su hábitat. Primero, analizamos las variaciones de abundancia a nivel especie-específico a través de las tres situaciones (SIN, CON y POST) en ambos ríos, con el fin de determinar el grado de sensibilidad de las distintas especies a este tipo de intervención.

Con el fin de evaluar cambios en las comunidades ícticas de las zonas potamales a través de los distintos estados de perturbación del cauce (SIN, CON y POST) se determinó la riqueza específica (S) y se estimó la diversidad, mediante los índices de Shannon en $\log 10\left(\mathrm{H}^{\prime}\right)$ y equidad de Pielou $\left(\mathrm{J}^{\prime}\right)$, (Magurran 1988). Para determinar si existieron cambios en los patrones de estructura comunitaria en las tres condiciones, SIN, CON y POST, se construyó una matriz de abundancia (número total de peces capturados en $100 \mathrm{~m}$ ) por sitio y muestreo, la cual fue transformada mediante raíz cuarta para disminuir el peso de las especies dominantes (Kreutzweiser et al. 2005). Para visualizar las relaciones de la comunidad de peces en las tres situaciones de intervención, se utilizó un análisis no métrico de escalamiento multidimensional (NMDS) basado en la matriz de similitud de BrayCurtis, utilizando el programa PRIMER-E Ltd v6.15 (Clarke \& Gorley 2005). Con el fin de estimar el grado de cambio de las comunidades en cada río en las tres situaciones analizadas, se midió la distancia (en $\mathrm{cm}$ ) entre puntos de un mismo río en el NMDS, estimando el cambio total como la sumatoria de los pasos entre las situaciones SIN, CON y POST. Este 
método es válido cuando el valor de estrés del ordenamiento tiene un valor cero o cercano a este (Clarke \& Warwick 2005). Con el fin de determinar la contribución de las distintas especies a los grupos ictiofaunísticos que caracterizaron el área en las tres condiciones ambientales SIN, CON y POST, se utilizó el procedimiento de Porcentajes de Similitud (SIMPER; v.6.15, Primer-E Ltd., Clarke \& Gorley 2005). Para estos fines, las estaciones P1 y P2 fueron consideradas como réplicas, dado que presentan las mismas características estructurales del hábitat y calidad del agua (Habit et al. 2007a). Finalmente, identificamos tres categorías de especies ícticas: especies resistentes, correspondientes a aquellas que recolonizan inmediatamente después de la perturbación y persisten después de ella; especies resilientes, correspondientes a aquellas que no permanecen después de la perturbación pero que posteriormente recolonizan el área impactada; $y$, especies sensibles, correspondientes a aquellas que se eliminan del área después del dragado y que no se recuperan.

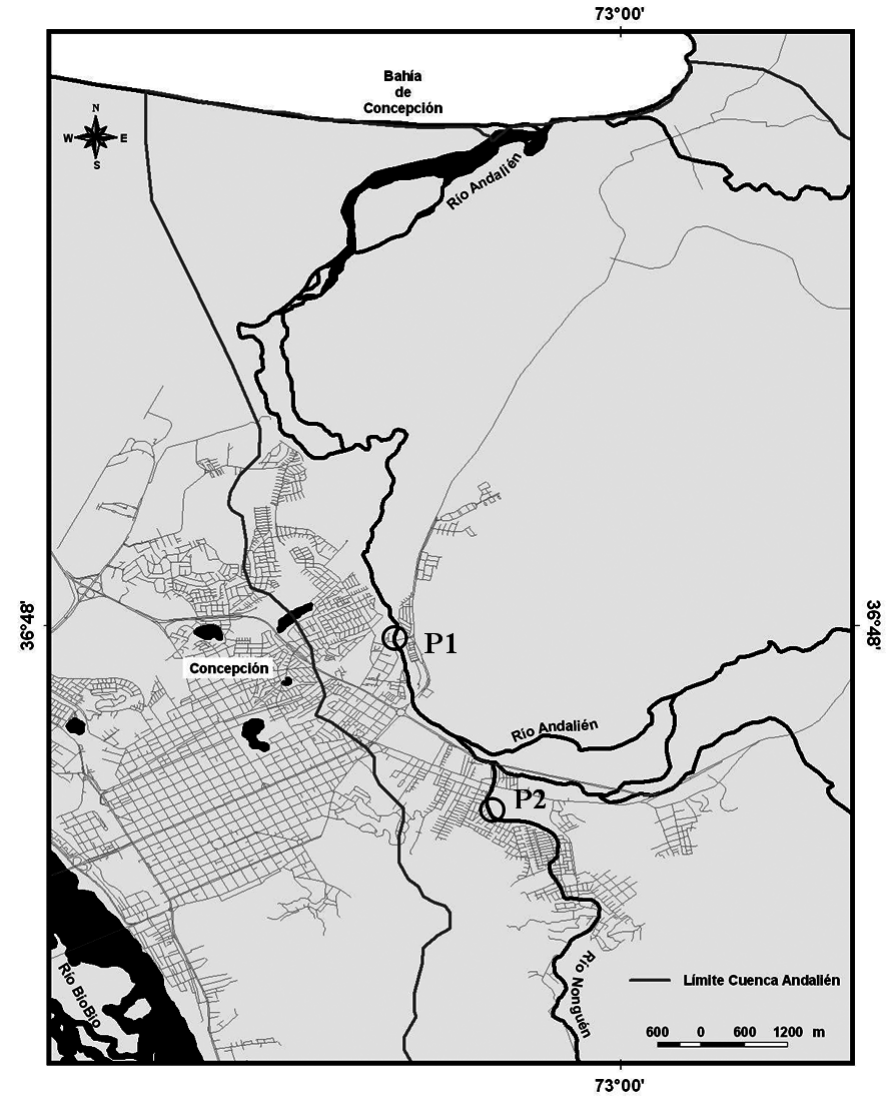

Figura 1. Esquema del área de estudio mostrando la cuenca del río Andalién en su límite con la cuenca del río Biobío y la extensión de la zona urbana de Concepción. Las estaciones de muestreo corresponden a P1 (río Andalién) y P2 (río Nonguén).

FIgURE 1. Study area showing the Andalién River Basin and its limit with Biobío River Basin and the urban zone of Concepción. Sampling stations correspond to P1 (Andalién River) and P2 (Nonguén River). 


\section{RESULTADOS}

Considerando los tres períodos de muestreo y las dos estaciones (P1 y P2), el área albergó un total de 15 especies, 10 nativas y 5 introducidas (Tabla I). De éstas, sólo Cheirodon galusdae (Eigenmann, 1928) fue capturada en las 3 situaciones (SIN, CON y POST, Fig. 2) y en ambos ríos, pero sin alcanzar la abundancia registrada en la situación SIN. Por el contrario, las especies nativas Nematogenys inermis
(Guichenot, 1848), Trichomycterus areolatus (Valenciennes, 1840) y Percichthys trucha (Valenciennes, 1833), más las especies introducidas Cichlasoma facetum (Jenyns, 1842), Gambusia holbrooki (Girard, 1854) y Carassius carassius (Linnaeus, 1758), no fueron capturadas en condiciones CON y POST. Bullockia maldonadoi (Eigenmann, 1928) se encontró en las tres condiciones en el río Nonguén, registrando una fuerte disminución de su abundancia en la situación CON,

Tabla I. Listado de especies de peces capturados en los tres períodos de muestreo, en ambas estaciones. El endemismo está referido a peces de agua dulce de la Provincia Biogeográfica Chilena sensu Dyer (2000). Las categorías de conservación están basadas en Campos et al. (1998). - Indica especies no clasificadas, correspondientes a especies introducidas en Chile.

Table I. List of fish species captured in the three sampling periods, in both stations. Endemism is referred to freshwater fish of the Chilean Province sensu Dyer (2000). Conservation categories are based on Campos et al. (1998). - Indicates unclassified species, corresponding to introduced species in Chile.

\begin{tabular}{|c|c|c|c|c|}
\hline Orden & Especie & Origen & Endémico & $\begin{array}{c}\text { Estado } \\
\text { de conservación }\end{array}$ \\
\hline Siluriformes & $\begin{array}{l}\text { Nematogenys inermis } \\
\text { (Guichenot, 1848) }\end{array}$ & Nativa & SI & En Peligro \\
\hline Siluriformes & $\begin{array}{l}\text { Trichomycterus areolatus } \\
\text { (Valenciennes, 1840) }\end{array}$ & Nativa & NO & Vulnerable \\
\hline Siluriformes & $\begin{array}{l}\text { Bullockia maldonadoi } \\
\text { (Eigenmann, 1928) }\end{array}$ & Nativa & SI & En Peligro \\
\hline Perciformes & $\begin{array}{l}\text { Percilia irwini } \\
\text { Eigenmann, } 1927\end{array}$ & Nativa & SI & En Peligro \\
\hline Perciformes & $\begin{array}{l}\text { Percichthys trucha } \\
\text { (Valenciennes, 1833) }\end{array}$ & Nativa & NO & Vulnerable \\
\hline Characiformes & $\begin{array}{l}\text { Cheirodon galusdae } \\
\text { Eigenmann, } 1928\end{array}$ & Nativa & SI & Vulnerable \\
\hline Petromizontiformes & $\begin{array}{l}\text { Geotria australis } \\
\text { Gray, } 1851\end{array}$ & Nativa & NO & Vulnerable \\
\hline Atheriniformes & $\begin{array}{l}\text { Basilichthys australis } \\
\text { Eigenmann, } 1928\end{array}$ & Nativa & SI & Vulnerable \\
\hline Atheriniformes & $\begin{array}{l}\text { Odontesthes (Cauque) mauleanum } \\
\text { (Steindachner, 1896) }\end{array}$ & Nativa & SI & Vulnerable \\
\hline Osmeriformes & $\begin{array}{l}\text { Galaxias maculatus } \\
\text { (Jenyns, 1842) }\end{array}$ & Nativa & NO & Vulnerable \\
\hline Cichlidae & $\begin{array}{l}\text { Cichlasoma facetum } \\
\text { (Jenyns, 1842) }\end{array}$ & Introducida & - & - \\
\hline Salmoniformes & $\begin{array}{l}\text { Oncorhynchus mykiss } \\
\text { (Walbaum, 1792) }\end{array}$ & Introducida & - & - \\
\hline Salmoniformes & $\begin{array}{l}\text { Salmo trutta } \\
\text { Linnaeus, } 1758\end{array}$ & Introducida & - & - \\
\hline Cyprinodontiformes & $\begin{array}{l}\text { Gambusia holbrooki } \\
\text { Girard, } 1854\end{array}$ & Introducida & - & - \\
\hline Cypriniformes & $\begin{array}{l}\text { Carassius carassius } \\
\text { Linnaeus, } 1758\end{array}$ & Introducida & - & - \\
\hline
\end{tabular}




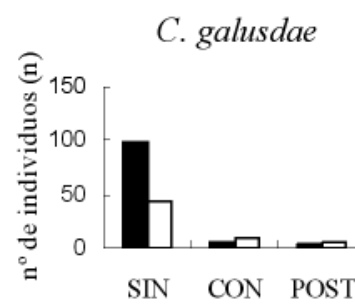

B. australis

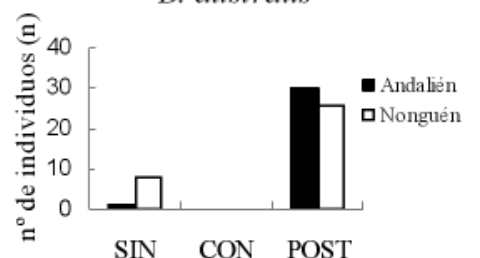

$N$. inermis

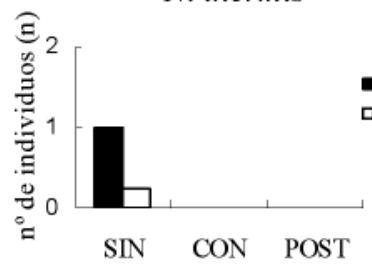

G. australis

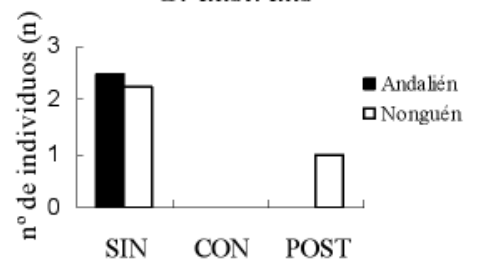

G. holbrooki

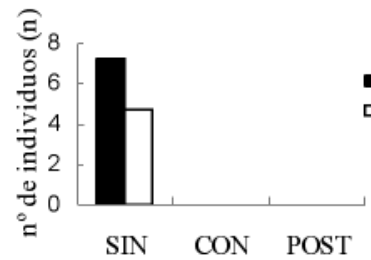

B. maldonadoi

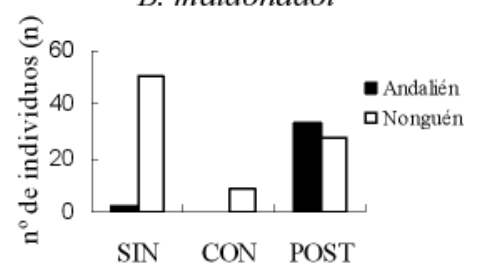

O. mykiss

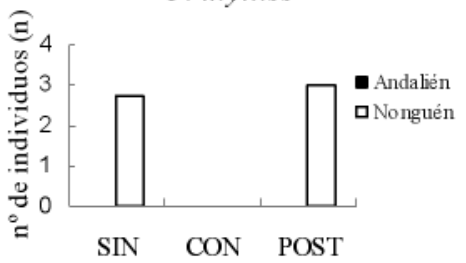

P. trucha

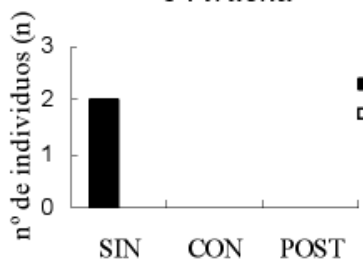

G. maculatus

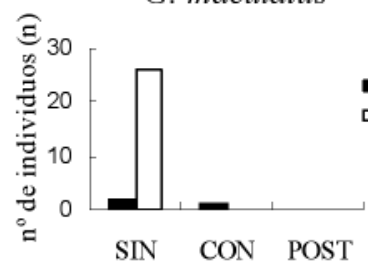

C. facetum

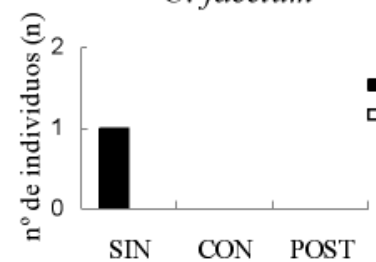

P. irwini

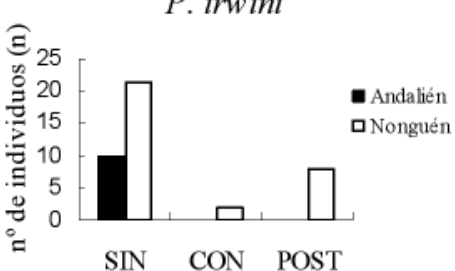

S. trutta

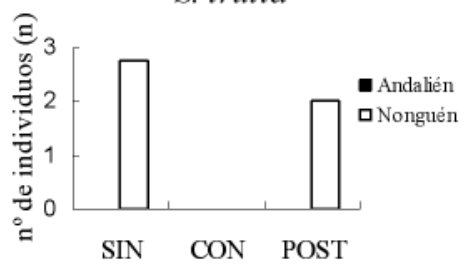

T. areolatus

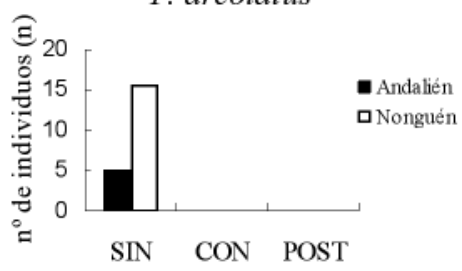

O. mauleanum

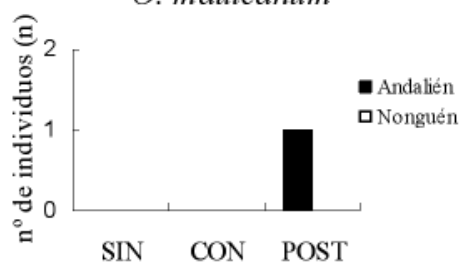

C. carassius

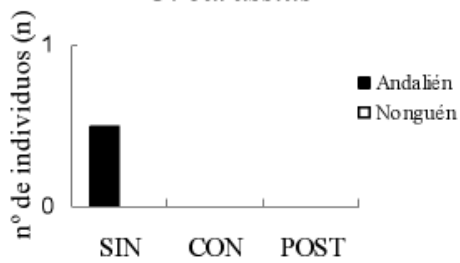

FIGURA 2. Abundancia especie-específica en la zona potamal de los ríos Andalién y Nonguén durante las tres situaciones SIN, CON y POST. Las escalas para cada especie son diferentes, dada la gran diferencia de abundancias entre ellas.

FIgURE 2. Species-specific abundances in the potamal zone of Andalién and Nonguén rivers during the three studied situations (SIN, CON and POST). Given difference in species abundances, scales are different. 
aumentando luego en POST, mientras que en el río Andalién a pesar que estuvo ausente en la situación CON, registró su mayor abundancia en POST. En la zona potamal del río Nonguén Percilia irwini (Eigenmann, 1927) registró un patrón similar a $B$. maldonadoi, mientras que en el río Andalién, no fue capturada en la condición CON y POST. Galaxias maculatus (Jenyns, 1842) y Geotria australis (Gray, 1851) bajaron drásticamente sus abundancias, siendo capturado sólo un ejemplar en la situación CON y POST respectivamente. Basilichthys australis (Eigenmann, 1928) estuvo presente en ambos ríos en condiciones SIN y POST, registrando su mayor abundancia en esta última condición, sin ser capturada en la situación CON en ambos ríos. Un comportamiento muy similar registraron las especies salmonídeas Oncorhynchus mykiss (Walbaum, 1792) y Salmo trutta (Linnaeus, 1758), exclusivamente en la estación del río Nonguén, observándose abundancias semejantes entre las condiciones SIN y POST. Además, en este estudio se capturó un único individuo de Odontesthes mauleanum (Steindachner, 1896) en el río Andalién durante el muestreo de 2007. Esta especie había sido descrita para el Andalién por Ruiz (1993), pero no detectada en los muestreos del 2004 y 2006. En relación a la condición SIN, las comunidades de peces de ambos ríos experimentaron una drástica disminución del número de especies $(\mathrm{S})$ y diversidad (índice de Shannon, $\mathrm{H}^{\prime}$ ), en la situación CON (Fig. 3). Ambos índices mostraron una recuperación en la condición POST pero sin alcanzar los valores originales (SIN). En tanto, la equidad (J') aumentó en las condiciones CON y POST, tanto en el río Andalién como en el Nonguén, dado que disminuyó la dominancia de B. maldonadoi y $C$. galusdae en el río Nonguén y de $C$. galusdae en el Andalién.

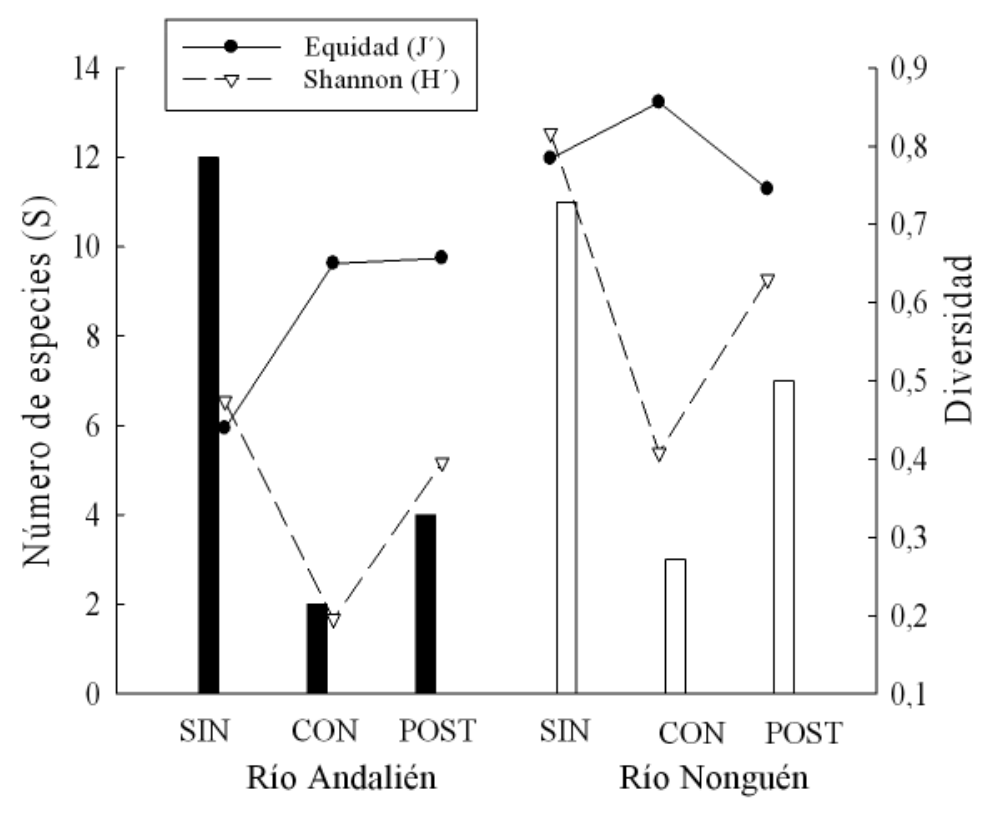

Figura 3. Riqueza de especies (S), Índice de Diversidad de Shannon ( $\left.\mathrm{H}^{\prime}\right)$ y Equidad de Pielou ( $\left.\mathrm{J}^{\prime}\right)$ de las zonas potamales de los ríos Andalién y Nonguén en las tres campañas de muestreo (SIN, CON y POST).

Figure 3. Species richness (S), Shannon Diversity Index $\left(\mathrm{H}^{\prime}\right)$ and Pielou's Evenness $\left(\mathrm{J}^{\prime}\right)$ of potamal zones of the Andalién and Nonguén rivers during the three sampling fieldtrips (SIN, CON y POST). 
El análisis de NMDS mostró una alta unidimensionalidad de los datos (estrés igual a cero) y cambios drásticos en los patrones comunitarios de la ictiofauna (Fig. 4). Tales cambios ocurrieron en la misma dirección en ambos ríos, pero con distintas magnitudes. El mayor cambio total de las comunidades ocurrió en el río Andalién (cambio total en el NMDS igual a 22,6 cm), en tanto que las comunidades del río Nonguén cambiaron un total de 11,8 cm. En ambos casos, se evidenció la recuperación de las comunidades, siendo la situación SIN más similar a la situación POST. Según el análisis de SIMPER, las comunidades de las zonas potamales de los ríos Andalién y Nonguén presentaban una alta similitud entre ellas $(70,2 \%)$, en la condición SIN, existiendo una contribución relativamente homogénea de las especies que las caracterizaban, correspondientes a $C$. galusdae
(20,3\% de contribución), P. irwini (14\%) y T. areolatus $(11,9 \%)$ (Tabla II). En la condición CON su similitud promedio disminuyó al $41,5 \%$, siendo C. galusdae la especie dominante con un $100 \%$ de contribución. Finalmente, en la condición POST se observó un valor de similitud intermedio $(64,2 \%)$, más cercano al de la situación SIN, pero un cambio en las especies que mayormente contribuyen a ella, correspondientes a $B$. maldonadoi $(39,1 \%), B$. australis $(38,4 \%)$ y C. galusdae $(22,4 \%)$.

La mayor disimilitud entre comparaciones pareadas de comunidades ocurrió entre las situaciones SIN y $\mathrm{CON}$, con un porcentaje de disimilitud igual al $68,2 \%$ (Tabla II). Las especies que más aportaron a esta disimilitud fueron $T$. areolatus y $G$. holbrooki, (11,8\% y $10,7 \%$, respectivamente), ya que ambas desaparecieron en la situación CON.

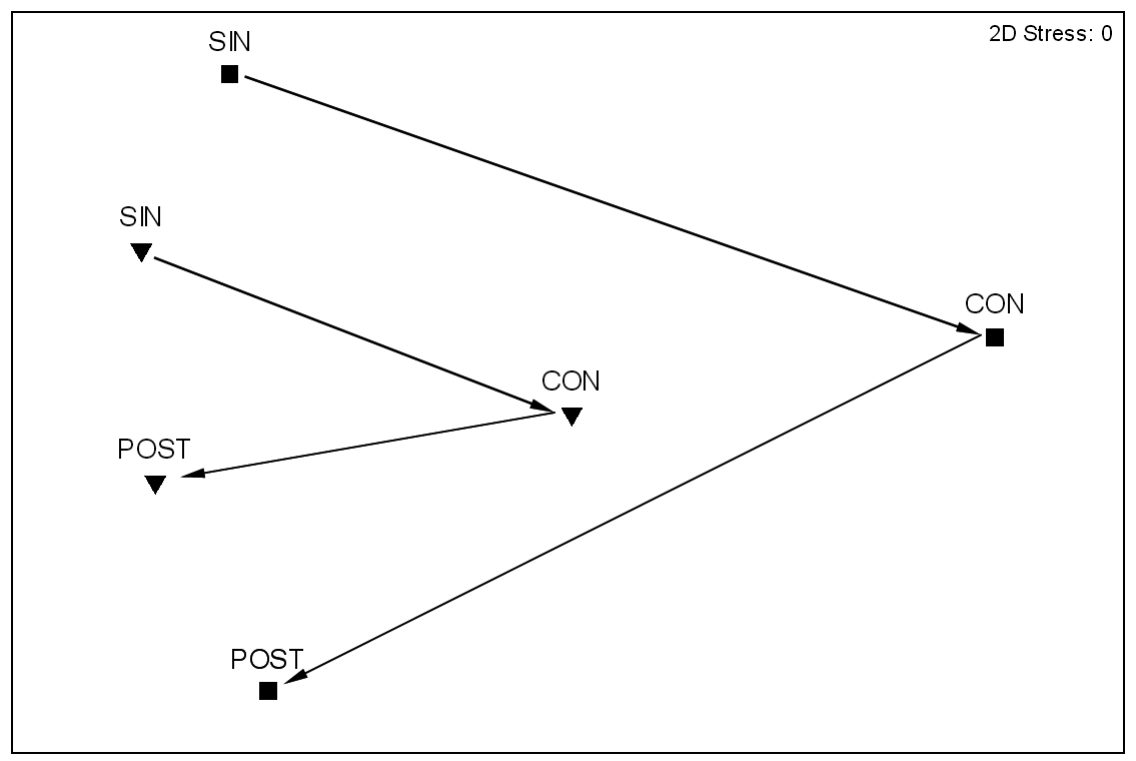

FIgURA 4. Escalamiento Multidimensional No Paramétrico (NMDS) en las tres situaciones estudiadas (SIN, CON Y POST), indicando la trayectoria de las comunidades en cada río. Cuadrados representan la estación P1 (río Andalién) y triángulos representan la estación P2 (río Nonguén).

FigURE 4. Non-Parametric Multidimensional Scaling (NMDS) for the three situations (SIN, CON Y POST), showing the communities trajectory in each river. Squares represent station P1 (Andalién River) and triangles represent station P2 (Nonguén River). 
Gayana 73(1), 2009

Los grupos ictiofaunísticos en las condiciones CON y POST mostraron una disimilitud levemente menor, de $60,8 \%$, siendo las especies B. australis y $B$. maldonadoi las de mayor contribución a esta disimilitud ( $30,9 \%$ y $21 \%$ respectivamente), debido a su mayor abundancia en la situación POST. Finalmente, las especies que más contribuyen a la disimilitud entre los grupos ictiofaunísticos de las condiciones SIN y POST (53,5\% de disimilitud) fueron T. areolatus (12\%), G. maculatus (11,8\%), G. holbrooki $(10,8 \%)$ y C. galusdae $(10,24 \%)$, todas las cuales disminuyeron sus abundancias en la condición POST.
De acuerdo a los resultados, definimos como sensibles a esta acción a las especies nativas Nematogenys inermis, Percichthys trucha, Galaxias maculatus, Trichomycterus areolatus y Geotria australis, y a las especies introducidas Carassius carassius, Cichlasoma facetum, Gambusia holbrooki. Como especies resilientes identificamos a Basilichthys australis, especie nativa, Salmo trutta y Oncorhynchus mykiss, especies introducidas. Finalmente, identificamos como especies resistentes a las especies nativas Cheirodon australe, Bullockia maldonadoi y Percilia irwini.

Tabla II. Análisis de Porcentajes de Similitud (SIMPER). Se muestran los valores de abundancia media (número de individuos) para los grupos ictiofaunísticos previo al dragado (SIN), con dragado (CON) y fase de recuperación (POST). En la comparación de pares, se muestran los valores de disimilitud media y en paréntesis los porcentajes $(\%)$ de contribución.

TABLE II. Percentage Similarity Analisys (SIMPER), showing mean abundance (number of individuals) for icthyofaunistics groups prior to dredged (SIN), dredged (CON) and recovery phase (POST). Pair wise comparison show average dissimilarity values and in parentheses the percentage $(\%)$ of contribution.

\begin{tabular}{lcccccc}
\hline Especie & \multicolumn{3}{c}{ Abundancia media } & \multicolumn{3}{c}{ Disimilitud media (contribución \%) } \\
& SIN & CON & POST & SIN / CON & SIN / POST & CON / POST \\
\hline N. inermis & 4,6 & 0 & 0 & $4,01(5,88)$ & $3,19(5,97)$ & - \\
O. mykiss & 2,4 & 0 & 1,5 & $2,82(4,14)$ & $2,42(4,52)$ & $4,46(7,33)$ \\
S. trutta & 0,9 & 0 & 1 & $2,82(4,14)$ & $2,37(4,42)$ & $4,03(6,62)$ \\
T. areolatus & 8 & 0 & 0 & $8,03(11,77)$ & $6,41(11,99)$ & - \\
B. maldonadoi & 21,95 & 4,5 & 30,5 & $6,22(9,11)$ & $2,75(5,13)$ & $12,77(20,98)$ \\
P. irwini & 8,5 & 1 & 4 & $6,44(9,45)$ & $4,36(8,15)$ & $6,36(10,46)$ \\
P. trucha & 0,45 & 0 & 0 & $2,93(4,29)$ & $2,31(4,32)$ & - \\
C. galusdae & 29,85 & 7,5 & 4 & $5,81(8,52)$ & $5,48(10,24)$ & - \\
G. australis & 1,35 & 0 & 0,5 & $5,78(8,47)$ & $2,9(5,41)$ & - \\
B. australis & 2,3 & 0 & 28 & $6,12(8,97)$ & $3,65(6,82)$ & $18,76(30,83)$ \\
G. holbrooki & 2,4 & 0 & 0 & $7,28(10,66)$ & $5,8(10,84)$ & - \\
C. facetum & 0,2 & 0 & 0 & - & - & - \\
C. carassius & 0,1 & 0 & 0 & - & - & - \\
G. maculatus & 5,6 & 0,5 & 0 & $5,43(7,96)$ & $6,3(11,78)$ & $4,44(7,29)$ \\
O. mauleanum & 0 & 0 & 0,5 & - & $2(3,74)$ & $4,75(7,8)$ \\
\hline
\end{tabular}


Respuesta de la comunidad de peces al dragado: Ortiz-SANDOval, J. ET AL.

\section{DISCUSION}

El dragado para la canalización de los cauces fluviales tiene un impacto negativo sobre la biota acuática, el cual es generado principalmente por la pérdida de la heterogeneidad espacial (Lau et al. 2006, Smiley \& Dibble 2008). En este estudio identificamos una respuesta de carácter temporal de la comunidad de peces al dragado del cauce, evidenciada por la recuperación parcial de las comunidades, asociada a la recuperación de los hábitats del río y, probablemente, de su biota asociada (macroinvertebrados). Esta recuperación se apreció en la rápida recolonización de la vegetación ribereña así como de la vegetación acuática sumergida y emergida en la situación postdragado, lo cual probablemente se vio acelerado por el alto contenido de materia orgánica en estos ríos (Habit et al. 2007a).

Un efecto negativo correspondió a la dominancia de una única especie (C. galusdae), inmediatamente después del dragado. En este sentido, el Characiforme $C$. galusdae fue la especie más resistente al dragado, lo que resulta consistente con lo descrito previamente en cuanto a su grado de tolerancia a diferentes tipos de perturbaciones (Victoriano 1997). Igualmente, identificamos a $B$. maldonadoi como tolerante, dado que es una especie de hábitos bentónicos que vive entre granos de sustrato fino (García \& Habit 2007), por lo que su hábitat es alterado directamente por el dragado. A pesar de ello, B. maldonadoi permaneció en la zona potamal del río Nonguén inmediatamente después del dragado y adicionalmente, aumentó su abundancia considerablemente en ambos ríos en relación a la situación previa. Este efecto de aumento de la abundancia puede deberse a una recolonización rápida desde sectores ubicados directamente aguas arriba. De igual forma, identificamos a $P$. irwini como especie resistente, aún cuando tuvo respuestas diferentes en los dos sistemas estudiados. En el río Nonguén, $P$. irwini estuvo presente en las tres situaciones estudiadas, en tanto que en el Andalién estuvo ausente en la condición con dragado. Esta respuesta diferencial parece estar modulada por las abundancias previas en ambos sistemas, ya que la población del potamón del río Nonguén era más abundante que en el río Andalién.

La única especie nativa que identificamos como resiliente correspondió al pejerrey chileno, $B$. australis, lo cual puede estar asociado a su alta capacidad de desplazamiento o vagilidad (Habit resultados no publicados), y a sus hábitos mas bien pelágicos (Campos et al. 1993). Otras especies resilientes resultaron ser los salmonídeos (introducidos), los que se caracterizan por ser altamente sensibles frente a alteraciones de calidad de agua, tales como aumento de sólidos suspendidos y turbidez (Börgstom 1973, James \& Graynoth 2002). Estos son los parámetros que resultan temporalmente alterados al realizar obras de dragado en un río. Por ello, es esperable que pueda existir una recolonización desde las zonas media y alta del río donde estas especies son más abundantes (Habit et al. 2007a).

Entre las especies identificadas como sensibles se encuentran 4 nativas: $N$. inermis, $T$. areolatus, $G$. maculatus y $P$. trucha; y 3 especies introducidas: $C$. facetum, G. holbrooki y C. carassius. De las especies nativas, $N$. inermis ha sido previamente mencionada como sensible en el río Biobío (Habit et al. 2006a), pero además es una especie rara, ya que naturalmente establece poblaciones de pequeño tamaño. De igual forma, $P$. trucha ha sido descrita como una especie que ha reducido sus abundancias en la zona potamal del río Biobío debido al efecto de distintas actividades antrópicas (Habit et al. 2006a), y que resulta sensible a la introducción de especies salmonídeas en sistemas lacustres (Gajardo \& Laikre 2003). En los sistemas estudiados, $P$. trucha forma parte del grupo ictiofaunístico de la zona transición-potamón, sin embargo, su mayor abundancia ocurre en la zona transicional (Habit et al. 2007a). En el caso de T. areolatus, es probable que la baja abundancia natural de esta especie en la zona potamal sea el factor que modula su pobre respuesta frente a esta perturbación. Sin embargo, este bagre nativo ha sido descrito como tolerante frente a otros tipos de perturbaciones, tales como operación de canales de riego (Habit \& Parra 2001) y centrales hidroeléctricas (Habit et al. 2007b). Naturalmente, las mayores abundancias de esta especie ocurren en la zona media de los ríos, con hábitats caracterizados por sustrato de gravilla y bolones, por lo que su presencia en el potamón es marginal a su distribución general en el río. Aún cuando se registró la presencia de Galaxias maculatus en el río Andalién en la situación con dragado fue identificada como sensible, ya que el hallazgo correspondió a un único individuo. Dado que esta especie se caracteriza por formar cardúmenes (Campos 1970, 1985, Campos et al. 
1993), la captura de un único individuo no es reflejo de una resistencia de la población a la perturbación. Una situación similar ocurre con la lamprea $G$. australis, especie sensible que es registrada en la situación post perturbación en el río Nonguén, con un individuo. Esta es una especie diádroma (Campos et al. 1993), cuyos estados larvales forman poblaciones dulceacuícolas (Jellyman \& Glova, 2002), las cuales eran poco abundantes en el área de estudio previo al dragado. Por ello, su respuesta estaría modulada por estos dos últimos factores (diadromía y abundancia). Dado que el hábitat natural de las especies introducidas identificadas como sensibles (C. facetum, G. holbrooki y C. carassius) corresponde a sistemas tropicales estables (Ross \& Doherty 1994), las condiciones en la cuenca del Andalién serían subóptimas para ellas, por lo cual la perturbación debida al dragado correspondería a una alteración de hábitat no tolerable para estas especies, mermando significativamente el tamaño de sus poblaciones.

La pérdida temporal de especies ícticas en el área de estudio posee un significado diferente a nivel especieespecífico. Desde el punto de vista de la conservación de la biodiversidad, la extirpación del área del bagre nativo $N$. inermis es un impacto negativo de alta relevancia, dado que es una especie en peligro de extinción (Campos et al. 1998), que ha reducido drásticamente su rango de distribución (Habit obs. pers.). Por el contrario, la pérdida de especies introducidas invasoras como C. facetum, G. holbrooki y $C$. carassius es un efecto positivo. A pesar que los efectos del dragado del cauce del río Andalién y Nonguén son altos en términos de diversidad íctica, éstos presentaron una naturaleza temporal, evidenciando una baja capacidad de resistencia, pero alta capacidad de resiliencia de la comunidad. La canalización del cauce con revestimiento, es una de las acciones que eventualmente podrían ejecutarse en el área, pero a diferencia del dragado la construcción de este tipo de obras implica la pérdida permanente de hábitats adecuados para el desarrollo de la biota acuática (Habit \& Parra 2001), lo que tendría un alto impacto negativo dada la valiosa fauna íctica que este sistema alberga.

\section{AGRADECIMIENTOS}

Agradecemos a Priscila Piedra y Jenna Trubschenck, quienes colaboraron en el muestreo. Este estudio fue financiado por los proyectos DIUC
204.310.041-1.0 de la Universidad de Concepción y por U.S. National Science Foundation OISE (PIRE) 0530267.

\section{BIBLIOGRAFIA}

Börgstom, R. 1973. The effect of increased water level fluctuation upon the brown trout population of Mar-Vann, a Norwegian reservoir. Norwegian Journal of Zoology. 21: 101-112.

CAmpos, H. 1970. Galaxias maculatus (Jenyns) en Chile, con especial referencia a su reproducción. Boletín del Museo de Historia Natural de Chile. 31: 5-20.

Campos, H. 1985. Distribution of the fishes in the Andean rivers in the South of Chile. Archiv fuer Hydrobiologie. 104: 169-191.

Campos, H., F. Alay, V. Ruiz \& J. Gavilán. 1993. Antecedentes biológicos de la fauna íctica presente en la hoya hidrográfica del río Biobío. En: Seminario Limnología y Evaluación de Impacto Ambiental (Eds. Faranda F, Parra O). Ediciones Centro EULA-Chile, Universidad de Concepción, Chile. 70-72.

Campos, H., G. Dazarola, B. Dyer, I. Fuentes, J.F. Gavilán, L. Huaquín, G. Martínez, R. MeléndeZ, G. Pequeño, F. Ponce, V.h. Ruiz, W. Siefeld, D. Soto, R. Vega \& I. Vila. 1998. Categorías de conservación de peces nativos de aguas continentales de Chile. Boletín del Museo Nacional de Historia Natural (Chile). 47: 101-122.

Clarke, K.R. \& R.N. Gorley. 2005. PRIMER v.6: User Manual / Tutorial. PRIMER-E Ltda., Playmouth, UK.

Clarke, K.R., R.M. Warwick, P.J. Somerfield \& R.N. GORLEY. 2005. Change in marine communities: an approach to statistical analysis and interpretation, 3rd edn. PRIMER-E Ltda, Plymouth, UK.

Dga: Dirección General De Aguas. 2004. Diagnostico y clasificación de los cuerpos de agua según objetivos de calidad. Cuenca del Río Biobío.

Duarte, W., R. Feito, C. Jara, C. Moreno \& A. Orellana. 1971. Ictiofauna del sistema hidrográfico del río Maipo. Boletín del Museo Nacional de Historia Natural de Chile. 32:227-268.

Dudgeon, D. 2000. Conservation of freshwater biodiversity in Oriental Asia: constraints, conflicts, and challenges to science and sustainability. Limnology. 1(3): 237-243.

DYER, B. 2000. Systematic review and biogeography of the freshwater fishes of Chile. Estudios Oceanológicos. 19:77-98.

Gajardo, G. \& L. Laikre. 2003. Chilean Aquaculture Boom Is Based on Exotic Salmon Resources: a Conservation Paradox. Conservation Biology. 17(4): 1173-1174.

García, A. \& E. Habit. 2007. Comparison of Qualitative and Pseudo-quantitative Approaches of Habitat Classification to Characterize Chilean Native Fish 
Respuesta de la comunidad de peces al dragado: OrTIZ-SANDOval, J. ET AL.

Species. 6th Symposium on Ecohydraulics, Christchurch, New Zealand.

Habit, E. \& O. Parra. 2001. Impactos ambientales de los canales de riego sobre la fauna de peces. Ambiente y Desarrollo (Chile). 17: 50-56.

Habit, E., P. Victoriano \& A. Rodriguez-ruiz. 2003. Variaciones espacio-temporales del ensamble de peces de un sistema fluvial de bajo orden del centro sur de Chile. Revista Chilena de Historia Natural. 76: 3-14.

Habit, E. \& P. Victoriano. 2005. Peces de agua dulce de la Cordillera de la Costa. En: Biodiversidad y Ecología de la Cordillera de la Costa de Chile (Eds. Smith-Ramírez C., Armesto, J., Valdovinos C.), pp. 374 - 389. Editorial Universitaria, Santiago, Chile.

Habit, E., M. Belk, C. Tuckfield \& O. Parra. 2006a. Response of the fish community to humaninduced changes in of the Biobío River in Chile. Freshwater Biology. 51:1-11.

Habit, E., B. Dyer \& I. Vila. 2006в. Estado del conocimiento de los peces dulceacuícolas de Chile. Gayana Zoología. 70 (1): 100-113.

Habit, E., M. BelK, P. Victoriano \& E. Jaque. 2007A. Spatio-temporal distribution patterns and conservation of fish assemblages in a Chilean coastal river. Biodiversity Conservation. 16: 3179-3191.

Habit, E., M. Belk \& O. Parra. 2007в. Response of the riverine fish community to the construction and operation of a diversion hydropower plant in central Chile. Aquatic Conservation: Marine and Freshwater Ecosystems. 17: 37-49.

ILlies, J. \& L. BotasaneAnu. 1963. Problemes et methodes de la clasification de la zonation ecologique des eaux courantes, considerees surtout du point de vue faunistique. Mitteilungen. Internationale Vereiningung fuer Theoretische und Angewandte Limnologie. 12: 1-57.

JAMES, G.D. \& E. GRAYNOTH. 2002. Influence of fluctuating lake levels and water quality on trout populations in littoral zones of New Zealand alpine lakes. New Zealand Journal of Marine and Freshwater Research. 36: 39-52.

JAQUE, E. 1994. Problemas ambientales en un área de expansión urbana. Cuenca del estero Nonguén. Concepción. Chile. Revista Geográfica de Chile Terra Australis. 39: 65-78.

Jellyman, D.J. \& G.J. Glova. 2002. Habitat use by juvenile lampreys (Geotria australis) in a large New Zealand river. New Zealand Journal of Marine and Freshwater Research. 36: 503-510
Kreutzweiser, D.P., S.S. CAPell \& K.P. Good. 2005. Macroinvertebrate community responses to selection logging in riparian and upland areas of headwater catchments in a northern hardwood forest. Journal of the North American Bentholgical Society. 24: 208-222.

LAU, J.K., T.E. LAUER \& M.L. WeinMan. 2006. Impacts of Channelization on Stream Habitats and Associated Fish Assemblages in East Central Indiana. The American Midland Naturalist. 156: 319-330.

Magurran, A. 1988. Ecological diversity and its measurement. Princeton University Press, New Jersey, $179 \mathrm{pp}$.

Niemeyer, H. \& P. Cereceda. 1984. Hidrografía. Geografía de Chile. Tomo VIII.

Pérez, L. \& C. Andaur. 2005. Ciudad imaginada, ciudad planificada. Planificación urbana de Los Ángeles (Chile) en el siglo XX. Scripta Nova, Revista electrónica de geografía y ciencias sociales. 9(194): 88.

Ross, S.T. \& T.A. DOHERTY. 1994. Short-term persistence and stability of barrier island fish assemblages. Estuarine, Coastal and Shelf Science. 38: 39-47.

Ruiz, V. 1993. Ictiofauna del río Andalién (Concepción, Chile). Gayana Zoología. 57(2): 109-278.

RuIz, V. 1996. Ictiofauna del río Laja (VIII Región, Chile): una evaluación preliminar. Boletín de la Sociedad de Biología de Concepción. 67: 15-21.

Smiley, P.C. \& E.D. Dibble. 2008. Influence of spatial resolution on assessing channelization impacts on fish and macroinvertebrate communities in a warmwater stream in the southeastern United States. Environmental Monitoring Assessment. 138: 17-29.

Soto, D., I. Arismendi, J. Gonzalez, J. Sanzana, F. Jara, C. Jara, E .Guzman \& A. Lara. 2006. Sur de Chile, país de truchas y salmones: patrones de invasión y amenazas para las especies nativas. Revista Chilena de Historia Natural. 79(1): 97-117.

Victoriano, P. 1997. Variabilidad genética en Cheirodon galusdae (Pisces, Characidae) en una cuenca hidrográfica, y su relación con factores ambientales. Tesis. Doctorado en Ciencias Mención Zoología. Facultad de Ciencias Naturales y Oceanográficas. Universidad de Concepción 230 pp.

VILA, I., L. Fuentes \& M. Contreras. 1999. Peces límnicos de Chile. Boletín del Museo Nacional de Historia Natural de Chile. 48: 61-75.

Welcomme, R. 1985. River fisheries. FAO Fisheries Technical Paper. $262: 1-318$. 